Check for updates

Cite this: Chem. Sci., 2017, 8, 8400

\title{
Bulk assembly of organic metal halide nanotubes $\uparrow$
}

\author{
Haoran Lin, ${ }^{a}$ Chenkun Zhou, ${ }^{a}$ Yu Tian, ${ }^{b}$ Tiglet Besara, (D) ${ }^{c}$ Jennifer Neu, ${ }^{c}$

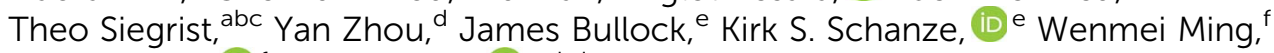 \\ Mao-Hua Du (D) ${ }^{f}$ and Biwu Ma (D) *abd
}

\begin{abstract}
The organic metal halide hybrids welcome a new member with a one-dimensional (1D) tubular structure. Herein we report the synthesis and characterization of a single crystalline bulk assembly of organic metal halide nanotubes, $\left(\mathrm{C}_{6} \mathrm{H}_{13} \mathrm{~N}_{4}\right)_{3} \mathrm{~Pb}_{2} \mathrm{Br}_{7}$. In a metal halide nanotube, six face-sharing metal halide dimers $\left(\mathrm{Pb}_{2} \mathrm{Br}_{9}{ }^{5-}\right)$ connect at the corners to form rings that extend in one dimension, of which the inside and outside surfaces are coated with protonated hexamethylenetetramine (HMTA) cations $\left(\mathrm{C}_{6} \mathrm{H}_{13} \mathrm{~N}_{4}{ }^{+}\right)$. This unique 1D tubular structure possesses highly localized electronic states with strong quantum confinement, resulting in the formation of self-trapped excitons that give strongly Stokes shifted broadband yellowish-white emission with a photoluminescence quantum efficiency (PLQE) of $\sim 7 \%$. Having realized single crystalline bulk assemblies of two-dimensional (2D) wells, 1D wires, and now 1D tubes using organic metal halide hybrids, our work significantly advances the research on bulk assemblies of quantum-confined materials.
\end{abstract}

Received 22nd August 2017
Accepted 16th October 2017

DOI: $10.1039 / \mathrm{c} 7 \mathrm{sc} 03675 \mathrm{~b}$

rsc.li/chemical-science a variety of optoelectronic devices from photovoltaic cells to light emitting diodes, photodetectors, and lasers. ${ }^{9-12}$ The exceptional structural tunability of this class of materials enables the formation of various types of crystal structures by using appropriate organic and inorganic components, ranging from three- (3D), to two- (2D), one- (1D), and zero-dimensional (0D) structures on the molecular level. ${ }^{13,14}$ In the 3D structure, small size cations, such as $\mathrm{Cs}^{+}$and $\mathrm{CH}_{3} \mathrm{NH}_{3}{ }^{+}$, fit into the cornersharing metal halide octahedrons $\left(\mathrm{MX}_{6}\right)$ to form a framework with the $3 \mathrm{D} \mathrm{MX}_{6}$ network. ${ }^{15,16}$ In the $2 \mathrm{D}$ structure, the $\mathrm{MX}_{6}$ octahedrons are connected in layered or corrugated sheets at the corners. ${ }^{17-19}$ In the $1 \mathrm{D}$ structure, the $\mathrm{MX}_{6}$ octahedrons are connected in a chain ${ }^{\mathbf{2 0 , 2 1}}$ and in the $0 \mathrm{D}$ structure, the $\mathrm{MX}_{6}$ octahedrons or assemblies of octahedrons are isolated from each other and surrounded by organic cations. ${ }^{22-24}$ These 2D, $1 \mathrm{D}$, and $0 \mathrm{D}$ hybrids can be considered as bulk assemblies of core-shell quantum confined materials, with 2D wells, 1D wires, and $0 \mathrm{D}$ individual metal halide octahedrons confined by organic ligands. The isolation between the building blocks in these low dimensional metal halide hybrids enables the bulk materials to exhibit the intrinsic properties of the individual building blocks. In other words, organic metal halide hybrids enable the properties of conventional individual nanostructures to be present in single crystalline bulk assemblies with defined periodicities. The structural versatility of this class of hybrid materials offers a vast parameter space to explore novel crystal structures exhibiting diverse properties.

Herein, we report for the first time an organic metal halide hybrid with 1D tubular structure. By using a proper organic cation, protonated hexamethylenetetramine (HMTA) $\left(\mathrm{C}_{6} \mathrm{H}_{13} \mathrm{~N}_{4}^{+}\right)$, we have been able to dictate the assembly of metal 
halides to form a bulk assembly of $1 \mathrm{D}$ metal halide nanotubes with a chemical formula of $\left(\mathrm{C}_{6} \mathrm{H}_{13} \mathrm{~N}_{4}\right)_{3} \mathrm{~Pb}_{2} \mathrm{Br}_{7}$. Due to the complete isolation and strong quantum confinement of individual metal halide nanotubes by wide band gap organic moieties, this nanotube-assembled material ( $\mathrm{HMTA})_{3} \mathrm{~Pb}_{2} \mathrm{Br}_{7}$ with 1D structure can exhibit the intrinsic properties of the individual metal halide nanotubes. A strongly Stokes shifted broadband yellowish-white emission peak at $580 \mathrm{~nm}$ with a large full width at half maximum (FWHM) of around $158 \mathrm{~nm}$ and a PLQE of around 7\% was realized as a result of exciton selftrapping in the metal halide frameworks..$^{25-33}$

\section{Results and discussion}

Single crystalline (HMTA) ${ }_{3} \mathrm{~Pb}_{2} \mathrm{Br}_{7}$ was prepared by slow solution diffusion of dichloromethane into dimethylformamide (DMF) precursor solutions containing $\mathrm{PbBr}_{2}$ and hexamethylenetetramine hydrobromide $\left(\mathrm{C}_{6} \mathrm{H}_{13} \mathrm{~N}_{4} \mathrm{Br}\right.$ ) (see experimental details in the ESI $\dagger$ ). Single crystal X-ray diffraction (SCXRD) was used to characterize the structure (Tables S1 and S2 $\dagger$ ). Fig. 1a shows large arrays of organic metal halide nanotubes, with an individual stripped metal halide nanotube shown in Fig. 1b and surrounded by organic cations as shown in Fig. 1c. The two basic building blocks for this organic metal halide hybrid are protonated HMTA $\left(\mathrm{C}_{6} \mathrm{H}_{13} \mathrm{~N}_{4}{ }^{+}\right)$(Fig. 1d) and face-sharing lead

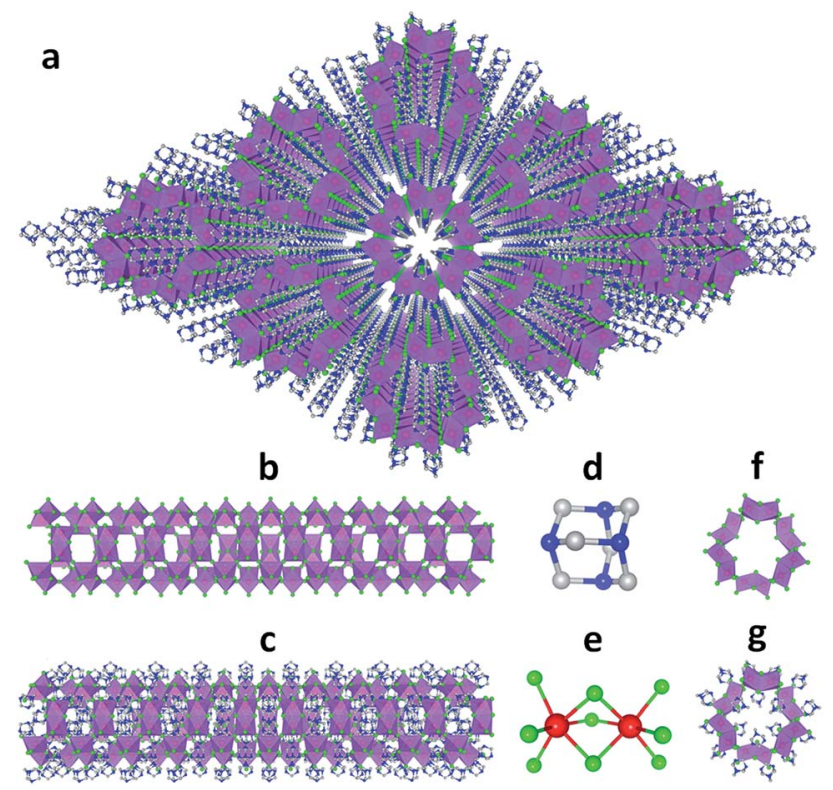

Fig. 1 (a) View of the structure of $(\mathrm{HMTA})_{3} \mathrm{~Pb}_{2} \mathrm{Br}_{7}$ (red: lead atoms; green: bromine atoms; blue: nitrogen atoms; gray: carbon atoms; purple polyhedra: $\mathrm{PbBr}_{6}$ octahedra and $\mathrm{Pb}_{2} \mathrm{Br}_{9}$ dimers; hydrogen atoms are hidden for clarity). (b) Side view of an individual lead bromide nanotube. (c) Side view of an individual lead bromide nanotube surrounded by organic cations. (d) Ball-and-stick model of an individual HMTA unit (hydrogen atoms are omitted). (e) Ball-and-stick model of an individual face-sharing lead bromide dimer $\mathrm{Pb}_{2} \mathrm{Br}_{9}$ with detailed bond lengths and bond angles listed in the ESI. $\dagger$ (f) Top view of a rugged ring formed by six lead bromide dimers. (g) Top view of a rugged metal halide ring coated with organic cations inside and outside. bromide dimers $\left(\mathrm{Pb}_{2} \mathrm{Br}_{9}{ }^{5-}\right)$ (Fig. 1e). In an individual nanotube, six lead bromide dimers connect at the corners to form rugged rings (Fig. 1f), with an inner radius of around $4 \AA$, that extend one dimensionally. The varied $\mathrm{Pb}-\mathrm{Br}$ bond lengths from $2.873 \AA$ to $3.163 \AA$ and $\mathrm{Br}-\mathrm{Pb}-\mathrm{Br}$ bond angles from $77.25^{\circ}$ to $113.15^{\circ}$ indicate that the present $\mathrm{PbBr}_{6}$ units are highly distorted and deviate from the typical octahedron configuration (Fig. S1, Tables S3 and S4 $\dagger$ ). In each of the "windows" formed by four connected dimers on an individual hollow nanotube, three protonated HMTA cations $\mathrm{C}_{6} \mathrm{H}_{13} \mathrm{~N}_{4}{ }^{+}$are anchored by coulombic interactions and hydrogen bonding, with one positioned inside the tube and the other two outside (Fig. 1g). In other words, individual negatively charged metal halide nanotubes are coated by positive organic cations on both the inside and the outside in a $1: 2$ ratio, resulting in an inorganic-organic coreshell nanotubular structure with an inner radius of around $1.5 \AA$ when taking the organic cations into account. These organic coated metal halide nanotubes further assemble into a hexagonal close packed array in the macroscopic crystal (Fig. 1a). The powder X-ray diffraction (PXRD) pattern of the ball-milled sample is identical to the simulated PXRD pattern from the single crystal structure (Fig. S2 $\dagger$ ), proving the reliability of the SCXRD results. This hybrid material exhibited moderate thermal stability without decomposition up to around $155^{\circ} \mathrm{C}$ in thermogravimetric analysis (TGA) (Fig. S3†). This organic metal halide hybrid with a unique nanotubular structure exhibits different properties from previously known organic metal halide hybrids, and could be of interest for various potential applications. Here we report its optical properties.
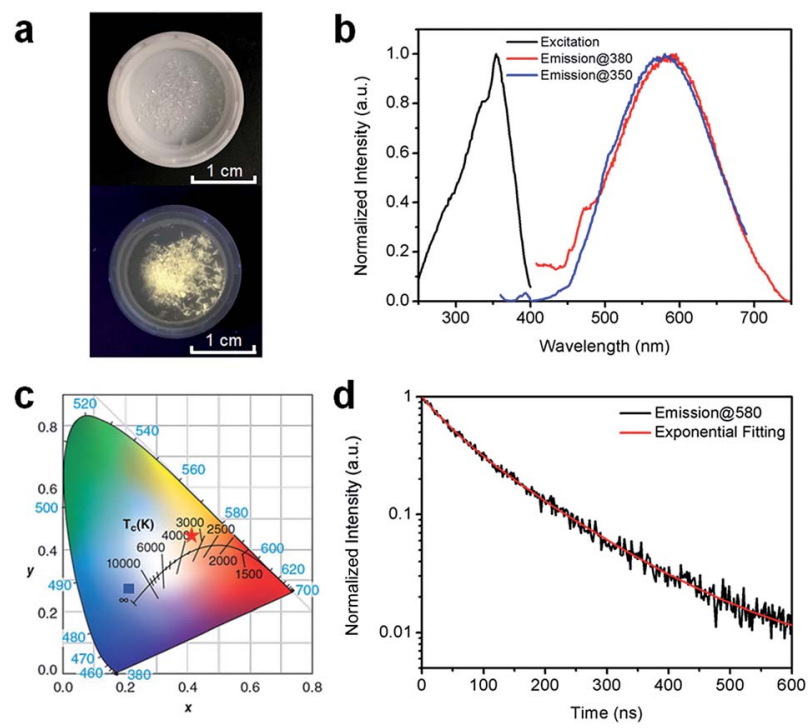

Fig. 2 (a) Images of (HMTA) ${ }_{3} \mathrm{~Pb}_{2} \mathrm{Br}_{7}$ crystals under ambient light (top) and UV light ( $365 \mathrm{~nm}$, bottom). (b) Excitation (black line, probed at 580 $\mathrm{nm}$ ) and emission (red line, excited at $380 \mathrm{~nm}$; blue line, excited at 350 $\mathrm{nm}$ ) spectra of (HMTA) ${ }_{3} \mathrm{~Pb}_{2} \mathrm{Br}_{7}$ crystals at room temperature. (c) $\mathrm{CIE}$ chromaticity coordinates of the bulk assembly of $1 \mathrm{D}$ lead bromide nanotubes in this work (red star), and the bulk assembly of 1D nanowires $\left(\mathrm{C}_{4} \mathrm{~N}_{2} \mathrm{H}_{14} \mathrm{PbBr}_{4}\right)$ (blue square) (ref. 21). (d) Time-resolved PL decay and fitting of $(\mathrm{HMTA})_{3} \mathrm{~Pb}_{2} \mathrm{Br}_{7}$ crystals (excited using a $365 \mathrm{~nm}$ diode laser, probed at $580 \mathrm{~nm}$ ) at room temperature. 
Fig. 2a shows the (HMTA) $)_{3} \mathrm{~Pb}_{2} \mathrm{Br}_{7}$ single crystals under ambient light and ultraviolet (UV) light (365 nm). The colorless crystals emit yellowish-white light or "warm" white under UV excitation with a reasonably good photostability (Fig. S4 $\dagger$ ), suggesting below-gap broadband emission with a large Stokes shift. As shown in Fig. 2b, (HMTA $)_{3} \mathrm{~Pb}_{2} \mathrm{Br}_{7}$ can indeed be excited by UV light from 250 to $400 \mathrm{~nm}$ and gives a broad emission peak at $580 \mathrm{~nm}$ that covers a wide range of the spectrum from 450 to $750 \mathrm{~nm}$ with a large FWHM of $158 \mathrm{~nm}$. We performed two emission scans under different excitation wavelengths $(350 \mathrm{~nm}$ and $380 \mathrm{~nm}$ ) to capture the whole emission spectrum. The position and shape of the two emission peaks are almost identical, indicating the excitation-independent emission property of this material. The Commission Internationale de l'Eclairage (CIE) chromaticity coordinates for this yellowishwhite emission are calculated to be $(0.42,0.45)$ (Fig. 2c), which represent a significant red-shift as compared to the bluish-white emission from a bulk assembly of 1D lead bromide nanowires $\left(\mathrm{C}_{4} \mathrm{~N}_{2} \mathrm{H}_{14} \mathrm{PbBr}_{4}\right) .{ }^{21}$ The PLQE of this material was measured to be around $7 \%$. This relatively low PLQE, as compared to those of organic lead bromide hybrids with 3D, 2Dlayered, and 1D-wire structures, is likely due to more nonradiative pathways present in this nanotube structure. The photoluminescence decay was characterized by time-resolved photoluminescence spectroscopy. As shown in Fig. 2d, biexponential fitting of the intensity-time curve gives an average lifetime of approximately 106 ns (the functional formula is specified in the ESI $\dagger$ ). Such a strongly Stokes shifted broadband emission with a relatively long lifetime is indeed similar to what has been observed in corrugated-2D and $1 \mathrm{D}$ metal halide perovskites. $^{21,25-33}$ It is therefore reasonable to attribute this non-Gaussian shaped broadband emission to the self-trapped excited states with multiple energy minimums, which are in thermally activated equilibrium at room temperature. Unlike emissions from both free excitons and self-trapped excited states in a bulk assembly of metal halide nanowires $\left(\mathrm{C}_{4} \mathrm{~N}_{2} \mathrm{H}_{14}\right.$ $\left.\mathrm{PbBr}_{4}\right){ }^{21}$ only self-trapped emissions were recorded for (HMTA $)_{3} \mathrm{~Pb}_{2} \mathrm{Br}_{7}$ (Fig. 2b), suggesting the absence of delocalized excitonic states due to the ultrafast exciton self-trapping in (HMTA $)_{3} \mathrm{~Pb}_{2} \mathrm{Br}_{7}$ at room temperature. This is not surprising if we compare the structure of $1 \mathrm{D}$ lead bromide nanotubes to that of nanowires. The electron coupling and molecular orbital interaction between the metal halide dimers via corner-sharing in 1D nanotubes are much weaker than those between the edge sharing metal halide octahedra in 1D nanowires. Therefore, the reduced conjugation in metal halide nanotubes produces more localized electronic states with stronger electron-phonon coupling, favoring the formation of self-trapped excitons.

To further confirm the origin of the broadband emission from the self-trapped excited states, we have characterized the temperature-dependent emission property of (HMTA $)_{3} \mathrm{~Pb}_{2} \mathrm{Br}_{7}$ by measuring its photoluminescence (PL) from $77 \mathrm{~K}$ to $300 \mathrm{~K}$ (Fig. 3a). At $77 \mathrm{~K}$, three emission peaks at $420 \mathrm{~nm}, 480 \mathrm{~nm}$ and $625 \mathrm{~nm}$ were observed. These emissions have different decay lifetimes as shown in Fig. $3 \mathrm{~b}$, which are estimated to be $7 \mu \mathrm{s}, 4.0$ $\mu \mathrm{s}$ and $1.8 \mu \mathrm{s}$ from the short wavelength to long wavelength, respectively (the functional formulas are specified in the ESI $\dagger$ ).
The long emission decay time is consistent with the typically observed slow spin-forbidden triplet exciton emission. Such distinct emission bands with different lifetimes at $77 \mathrm{~K}$ clearly indicate that multiple excited states are generated upon photoexcitation, which is consistent with the inherent properties of exciton self-trapping in metal halide perovskites. At low temperature $(77 \mathrm{~K})$, the trapped excitons could not overcome the energy barriers between different trapped excited states. In other words, the thermally activated equilibrium is likely suspended. As a result, trapped excited states with higher energy but faster self-trapping processes are kinetically populated and give distinguishable emissions at $420 \mathrm{~nm}$ and $480 \mathrm{~nm}$. The formation of these self-trapped excitons with higher energy may involve elongation of the $\mathrm{Pb}-\mathrm{Br}$ bonds within a $\mathrm{PbBr}_{6}$ octahedron. ${ }^{34}$ During the warming process, the peak intensities at $420 \mathrm{~nm}$ and $480 \mathrm{~nm}$ decreased while the peak intensity at $625 \mathrm{~nm}$ increased significantly. This phenomenon indicates that when the temperature rises, the trapped excitons can obtain enough thermal energy to overcome the energy barriers between different trapped states, forming a thermal equilibrium. Therefore, the emission at room temperature is mostly from the self-trapped states which have the lowest energy among all of the excited states. The formation of these trapped states with lower energy may originate from stronger lattice distortion, which requires a larger kinetic barrier. ${ }^{35}$ For clarity, the photoluminescence mechanism for this bulk assembly of metal halide nanotubes is depicted in the configuration coordinate diagram given in Fig. $3 \mathrm{c}$ and $d$. Upon UV excitation, the lead bromide nanotubes are excited to the high energy excited states, which undergo ultrafast exciton self-trapping with the formation of multiple excited states that give multiple emission bands at $77 \mathrm{~K}$ without thermally activated equilibrium, and strongly Stokes shifted broadband photoluminescence at room temperature due to thermally activated equilibrium. The observation of multiple exciton emissions with strong temperature dependence resembles the emissions of $\mathrm{ns}^{2}$ ions in alkali halides. ${ }^{36}$

To gain deeper insight into the physical mechanism underpinning the photophysics of this bulk assembly of metal halide nanotubes, we performed density functional theory (DFT) calculations. The calculated band structure of (HTMA) ${ }_{3} \mathrm{~Pb}_{2} \mathrm{Br}_{7}$ (Fig. 4a) shows a direct band gap at the $\Gamma$ point. The calculated band gap is $2.37 \mathrm{eV}$ at the Perdew-Burke-Ernzerhof (PBE) level, which is expected to be underestimated due to the well-known band gap error of DFT. The conduction and the valence bands are flat on the plane perpendicular to the axis of the tube, indicating negligible inter-tubular interaction. Along the axis of the tube, the band dispersion is still small, reflecting the weakened $\mathrm{Pb}-\mathrm{Br}$ hybridization due to the distortion of the $\mathrm{PbBr}_{6}$ octahedral structure. The narrow bands near the band edges and the soft lattice of ( $\mathrm{HTMA})_{3} \mathrm{~Pb}_{2} \mathrm{Br}_{7}$ should favor charge localization and the formation of self-trapped excitons, ${ }^{37-39}$ which is consistent with the absence of free exciton emission at room temperature. The top of the valence band is mostly made up of $\mathrm{Br}-4 \mathrm{p}$ states but has significant $\mathrm{Pb}-6 \mathrm{~s}$ character, while the bottom of the conduction band is dominated by $\mathrm{Pb}-6 \mathrm{p}$ states as shown by the projected density of states (DOS) in Fig. 4b. Thus, 

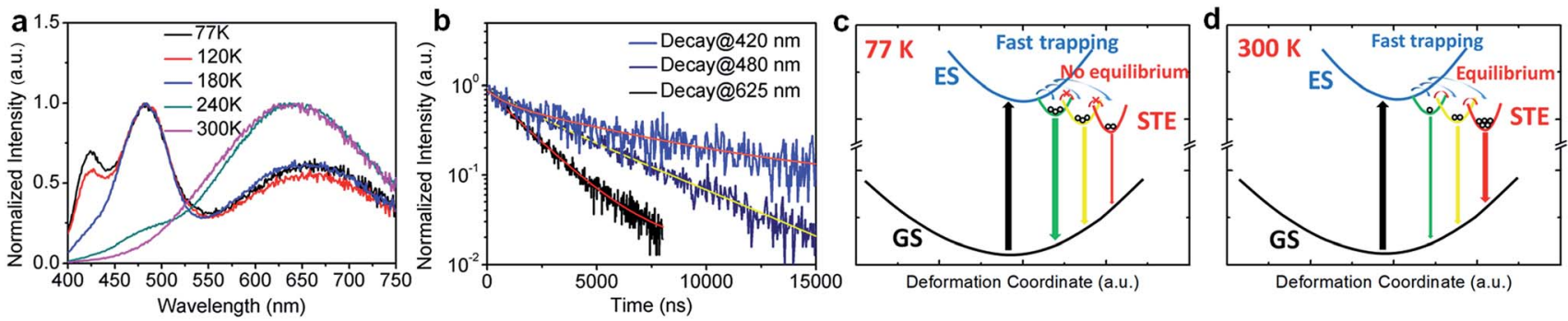

Fig. 3 (a) Temperature-dependent PL spectrum of (HMTA) ${ }_{3} \mathrm{~Pb}_{2} \mathrm{Br}_{7}$ crystals (excited at $380 \mathrm{~nm}$ ) from $77 \mathrm{~K}$ to $300 \mathrm{~K}$. (b) The emission decays at different wavelengths at $77 \mathrm{~K}$ and their multi-exponential fitting curves (excited using a $365 \mathrm{~nm}$ diode pulse laser, probed at $420 \mathrm{~nm}, 480 \mathrm{~nm}$ and $625 \mathrm{~nm})$. (c-d) Proposed energy diagrams and excited state dynamics of ( $\mathrm{HMTA})_{3} \mathrm{~Pb}_{2} \mathrm{Br}_{7}$ at $77 \mathrm{~K}$ and at $300 \mathrm{~K}$ : GS $=$ ground state, ES $=$ excited state, STE = self-trapped states, and the straight and curved arrows represent optical and energy relaxation/transfer transitions, respectively. Circles represent self-trapped excitons.
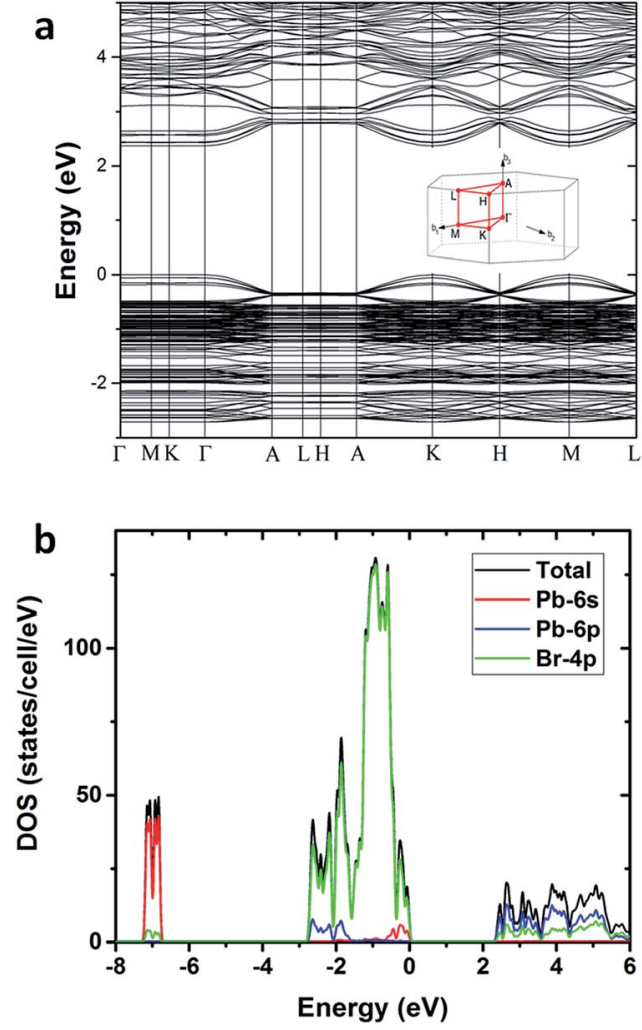

Fig. 4 (a) Calculated band structure of $(\mathrm{HTMA})_{3} \mathrm{~Pb}_{2} \mathrm{Br}_{7}$. (b) Projected density of states of $(\mathrm{HTMA})_{3} \mathrm{~Pb}_{2} \mathrm{Br}_{7}$.

excitons should be localized on the $\mathrm{Pb}^{2+}$ ions and the emission is likely due to the $\mathrm{Pb} 6 \mathrm{p}-6 \mathrm{~s}$ transition.

\section{Conclusions}

In summary, we have prepared for the first time a novel single crystalline organic metal halide hybrid containing arrays of metal halide nanotubes via a simple bottom up solution selfassembly process. Our work bridges the research of organic metal halide hybrids with functional nanotube materials. The excitement of our work lies not only in the specific achievements, but also in what it represents in terms of new opportunities for organic metal halide hybrids beyond conventional structures. On-going research aims to explore the optical, electronic, and other properties of this new class of organic metal halide hybrids with tubular structures, and develop theories to predict and guide the synthesis of possible organic metal halide frameworks. Efforts to improve the photoluminescence properties of such nanotube assemblies by making more rigid structures are also underway.

\section{Conflicts of interest}

There are no conflicts to declare.

\section{Acknowledgements}

The authors acknowledge the Florida State University for the support through the Energy and Materials Initiative and GAP Commercialization Grant Program. C. Z., Y. T., and B. M. acknowledge funding support from the National Science Foundation (NSF) (DMR-1709116). J. N. and T. S. acknowledge the funding from the NSF (DMR-1606952). The work at the NHMFL was supported by the NSF Cooperative Agreement No. DMR-1157490, the State of Florida and the Department of Energy. The work at ORNL was supported by the Department of Energy, Office of Science, Basic Energy Sciences, Materials Sciences and Engineering Division. The authors thank Dr Kenneth Hanson at FSU for providing access to a spectrophotometer and Dr Peter Djurovich at the University of Southern California for the help with PLQE measurement.

\section{Notes and references}

1 S. Iijima, Nature, 1991, 354, 56-58.

2 Y. X. Jiang, A. Lee, J. Y. Chen, M. Cadene, B. T. Chait and R. MacKinnon, Nature, 2002, 417, 515-522.

3 F. N. Dai, H. Y. He and D. F. Sun, J. Am. Chem. Soc., 2008, 130, 14064-14065.

4 Y. G. Huang, F. L. Jiang, D. Q. Yuan, M. Y. Wu, Q. Gao, W. Wei and M. C. Hong, Cryst. Growth Des., 2008, 8, 166-168. 
5 T. T. Luo, H. C. Wu, Y. C. Jao, S. M. Huang, T. W. Tseng, Y. S. Wen, G. H. Lee, S. M. Peng and K. L. Lu, Angew. Chem., Int. Ed., 2009, 48, 9461-9464.

6 Y. Q. Jiao, H. Y. Zang, X. L. Wang, E. L. Zhou, B. Q. Song, C. G. Wang, K. Z. Shao and Z. M. Su, Chem. Commun., 2015, 51, 11313-11316.

7 G. Z. Shen, Y. Bando and D. Golberg, Int. J. Nanotechnol., 2007, 4, 730-749.

8 P. Thanasekaran, T. T. Luo, C. H. Lee and K. L. Lu, J. Mater. Chem., 2011, 21, 13140-13149.

9 A. Kojima, K. Teshima, Y. Shirai and T. Miyasaka, J. Am. Chem. Soc., 2009, 131, 6050-6051.

10 Z. K. Tan, R. S. Moghaddam, M. L. Lai, P. Docampo, R. Higler, F. Deschler, M. Price, A. Sadhanala, L. M. Pazos, D. Credgington, F. Hanusch, T. Bein, H. J. Snaith and R. H. Friend, Nat. Nanotechnol., 2014, 9, 687-692.

11 G. C. Xing, N. Mathews, S. S. Lim, N. Yantara, X. F. Liu, D. Sabba, M. Gratzel, S. Mhaisalkar and T. C. Sum, Nat. Mater., 2014, 13, 476-480.

12 X. Hu, X. D. Zhang, L. Liang, J. Bao, S. Li, W. L. Yang and Y. Xie, Adv. Funct. Mater., 2014, 24, 7373-7380.

13 D. B. Mitzi, J. Chem. Soc., Dalton Trans., 2001, 1-12.

14 B. Saparov and D. B. Mitzi, Chem. Rev., 2016, 116, 4558-4596. 15 J. S. Manser, J. A. Christians and P. V. Kamat, Chem. Rev., 2016, 116, 12956-13008.

16 J. Berry, T. Buonassisi, D. A. Egger, G. Hodes, L. Kronik, Y. L. Loo, I. Lubomirsky, S. R. Marder, Y. Mastai, J. S. Miller, D. B. Mitzi, Y. Paz, A. M. Rappe, I. Riess, B. Rybtchinski, O. Stafsudd, V. Stevanovic, M. F. Toney, D. Zitoun, A. Kahn, D. Ginley and D. Cahen, Adv. Mater., 2015, 27, 5102-5112.

17 C. Huo, B. Cai, Z. Yuan, B. Ma and H. Zeng, Small Methods, 2017, 1, 1600018.

18 D. B. Mitzi, S. Wang, C. A. Feild, C. A. Chess and A. M. Guloy, Science, 1995, 267, 1473-1476.

19 L. T. Dou, A. B. Wong, Y. Yu, M. L. Lai, N. Kornienko, S. W. Eaton, A. Fu, C. G. Bischak, J. Ma, T. N. Ding, N. S. Ginsberg, L. W. Wang, A. P. Alivisatos and P. D. Yang, Science, 2015, 349, 1518-1521.

20 S. M. Wang, D. B. Mitzi, C. A. Feild and A. Guloy, J. Am. Chem. Soc., 1995, 117, 5297-5302.

21 Z. Yuan, C. Zhou, Y. Shu, Y. Tian, J. Messier, J. Wang, L. Burgt, K. Kountouriotis, Y. Xin, E. Holt, K. S. Schanze, R. Clark, T. Siegrist and B. Ma, Nat. Commun., 2017, 8, 14051.
22 C. Zhou, Z. Yuan, Y. Tian, H. Lin, R. Clark, B. Chen, L. Burgt, J. Wang, K. Hanson, Q. Meisner, J. Neu, T. Besara, T. Siegrist, E. Lambers, P. Djurovich and B. Ma, 2017, arXiv, 1702.07200.

23 B. R. Vincent, K. N. Robertson, T. S. Cameron and O. Knop, Can. J. Chem., 1987, 65, 1042-1046.

24 C. Zhou, Y. Tian, M. Wang, A. Rose, T. Besara, N. K. Doyle, Z. Yuan, J. C. Wang, R. Clark, Y. Hu, T. Siegrist, S. Lin and B. Ma, Angew. Chem., Int. Ed., 2017, 56, 9018-9022.

25 E. R. Dohner, A. Jaffe, L. R. Bradshaw and H. I. Karunadasa, J. Am. Chem. Soc., 2014, 136, 13154-13157.

26 E. R. Dohner, E. T. Hoke and H. I. Karunadasa, J. Am. Chem. Soc., 2014, 136, 1718-1721.

27 A. Yangui, D. Garrot, J. S. Lauret, A. Lusson, G. Bouchez, E. Deleporte, S. Pillet, E. E. Bendeif, M. Castro, S. Triki, Y. Abid and K. Boukheddaden, J. Phys. Chem. C, 2015, 119, 23638-23647.

28 T. Hu, M. D. Smith, E. R. Dohner, M. J. Sher, X. X. Wu, M. T. Trinh, A. Fisher, J. Corbett, X. Y. Zhu, H. I. Karunadasa and A. M. Lindenberg, J. Phys. Chem. Lett., 2016, 7, 2258-2263.

29 Z. Yuan, C. K. Zhou, J. Messier, Y. Tian, Y. Shu, J. M. Wang, Y. Xin and B. W. Ma, Adv. Opt. Mater., 2016, 4, 2009-2015.

30 J. Yin, H. Li, D. Cortecchia, C. Soci and J. L. Bredas, ACS Energy Lett., 2017, 2, 417-423.

31 L. L. Mao, Y. L. Wu, C. C. Stoumpos, M. R. Wasielewski and M. G. Kanatzidis, J. Am. Chem. Soc., 2017, 139, 5210-5215.

32 D. Cortecchia, J. Yin, A. Bruno, S. Z. A. Lo, G. G. Gurzadyan, S. Mhaisalkar, J. L. Bredas and C. Soci, J. Mater. Chem. C, 2017, 5, 2771-2780.

33 D. Cortecchia, S. Neutzner, A. R. S. Kandada, E. Mosconi, D. Meggiolaro, F. De Angelis, C. Soci and A. Petrozza, J. Am. Chem. Soc., 2017, 139, 39-42.

34 J. Yin, H. Li, D. Cortecchia, C. Soci and J.-L. Brédas, ACS Energy Lett., 2017, 2, 417-423.

35 B. Kang and K. Biswas, J. Phys. Chem. C, 2016, 120, 1218712195.

36 P. W. M. Jacobs, J. Phys. Chem. Solids, 1991, 52, 35-67.

37 K. Biswas and M. H. Du, Phys. Rev. B: Condens. Matter Mater. Phys., 2012, 86, 014102.

38 H. L. Shi and M. H. Du, Phys. Rev. Appl., 2015, 3, 014004.

39 M. H. Du, ECS J. Solid State Sci. Technol., 2016, 5, R3007R3018. 